\title{
A Framework for Ranking of WSNs Performance Based on Cloud Model and Entropy Weight
}

\author{
Xiaojun $\mathrm{Yu}$ \\ School of Computer Engineering, Jiangsu University of Technology \\ Changzhou 213001, Jiangsu, China \\ yxj_aqi@163.com
}

\begin{abstract}
Wireless Sensor Network (WSN) has become one of the cutting edge technologies, more attention has been paid to the further development and more applications emerging, WSNs is no longer confined to the traditional area. Due to the charadters of dynami, distribution, resource constrained, uncertainty, complexity and heterogeneity, we need whole evaluation framework of WSNs performance, which can allow user choose and monitor the operation state. In this paper, we construct WSNPI architecture to rank and observe WSNs, which includes WSNPI Broker, Monitoring and WSNs Catalogue. We offer a more comprehensive evaluation index system including accountability, agility cost security and privacy, then build a cloud model and entropy weight method to evaluate WSNs performance. Finally, the proposed method has been serviced to complex simulation and the results have indicated that the model is objective and effective. The firs part is the research status and related problems. The second part is the establishmentof an evaluation framework and model. The last part is the numerical example analysis and conclusion
\end{abstract}

Keywords: WSNs, non-equilibrium statisical mechanics method, performance evaluation

\section{Introduction}

Wireless Sensor Network (WSN) has been one of the cutting edge technologies for low power wireless communication $[1,2]$. Last several years, with the rapid development of embedded computing technology, modern network, wireless communication technology, wireless sensor network has a very vast application prospect and potential utility value, which integrates a large number of relative technology together [3, 4]. In most of WSN applications, a large number of sensor nodes are deployed to gather data based on application domains [5] [6]. WSNs opens up a wealth of opportunities for different applications, such as agriculture, city management, environmental monitoring, health management, emergency rescue and disaster relief, anti-terrorism, remote control of hazardous area and so on [7-9]. More attention has been paid to the further development and more applications emerging, WSNs is nolonger confined to the traditional area, for example WSNs can currently operate heating, ventilating, and air-conditioning and lighting systems efficiently, moreover improve the comfort of occupants while reducing energy consumption, and additionally augment the safety and security systems [10]. In the context of WSNs, a number of empirical studies present network measurement results in some aspects, with emphasis on understanding this complex and non-ideal behavior of low power wireless communications. Thus, the emergence of wireless sensor networks will bring great change to human society.

Despite the apparent ease with which people can deploy wireless sensors and actuators, engineers and operators still have concerns and questions relating to the use of wireless 
technology [1, 12]. Because WSNs is dynamic, distribution, resource constrained, uncertainty, complexity and heterogeneity, meanwhile different WSNs applications have unique characteristics and requirements in the respective field, which both urge us to further research, especially performance evaluation of WSNs. In the new distributed autonomous environment, the requirement for real-time and reliability of the signal transmission is very high $[13,14]$. In order to study the effect of network delay on control performance, we must put forward the corresponding evaluation method to test and analyze the performance of WSNs. Based on the analysis mentioned above, we can obtain the overall network performance, and then evaluate the identification and acceptance of an existing network[15] [16]. As for a planned network, we also greatly depend on the evaluation of the scheme using the performance of WSNs, in order to choose the suitable network structure and the deployment of network nodes.

Lots of research works have been conducted on various aspects of WSN including protocol and architecture, network topology, routing and application prospects, power conservation etc., [17-20]. Network performance supported in WSNs is still remained as an open field of research from diverse perspectives. Network performanee is interpreted by different technical communities in different ways. At present, there is no Jonger a unified standard and rank framework, according to the performance index of WSNs. Only some related researches for the parameters of WSNs performance, which are speed, accuracy, dependability, availability and so on [21, 22]. Gholami, N. Cai emphasized the temporal performance dynamics of wireless links and provided important findings about such phenomenon [23]. Selcuk had proposed evaluating WSNs performance from a global or overall angle [24]. Varadharajan evaluated WSNs performance binding relative comparison method and variation coefficient method together [25]. To evaluate the performance of WSNs scientifically, we need a new framewok and rank method to determine the weight of different index, and fully reflect the objectivity and accuacy of WSNs performance.

In summary, current research of WSNs performance evaluation is in its infancy, there is considerable problem space to explore and solve. On one hand, WSNs performance indexes usually are very limited $[26,27]$, which neglects the other factors which have huge effect on WSNs performance Qn the other hand, we need a whole evaluation framework of WSNs performance, which can help uses choose and monitor the operation state. At the same time, indexes have crossover influence and weight is not taken into account [28]. Therefore, with considering the complex characters of WSNs in different applications, there are three measures to take, Firstly, construct WSNPI architecture to rank and observe WSNs, which includes WSNPI Broker, Monitoring and WSNs Catalogue. We propose a more comprehensive evaluation index system including accountability, agility, cost, actual performance, security and privacy. Secondly, we create a cloud model and entropy weight method to evaluate WSNs performance. Finally, we apply Opnet to collect data of WSNs, and the proposed method has been used in complex simulation, and the results have indicated that the model is unbiased and effective.

\section{WSNPI and WSNPI System Architecture}

Network performance reflects the quality of WSN to provide communications services, that is the embodiment of the characteristics of itself, can be composed of a series of index definitions and descriptions [29, 30]. IP Performance Metrics Working Group had put forward the principles and framework about definition of performance indicators, and defined some indicators of assessment of IP network data transmission services quality, performance and reliability, such as connectivity, one-way delay, loop back delay, packet loss rate, delay 
variation. Study Group 13 proposed and defined 4 parameters to evaluate IP packet transmission performance, such as speed, accuracy, dependability, availability. It also sets a series of performance indicators, such as transmission delay, delay variation, packet error rate, packet loss rate, false IP packet rate, packet throughput, the throughput of bytes. However, users have various kinds of requirements in diverse applications, and the rank index is also different. In the article, we put forward a whole new framework to evaluate WSNs performance which is called WSNPI architecture.

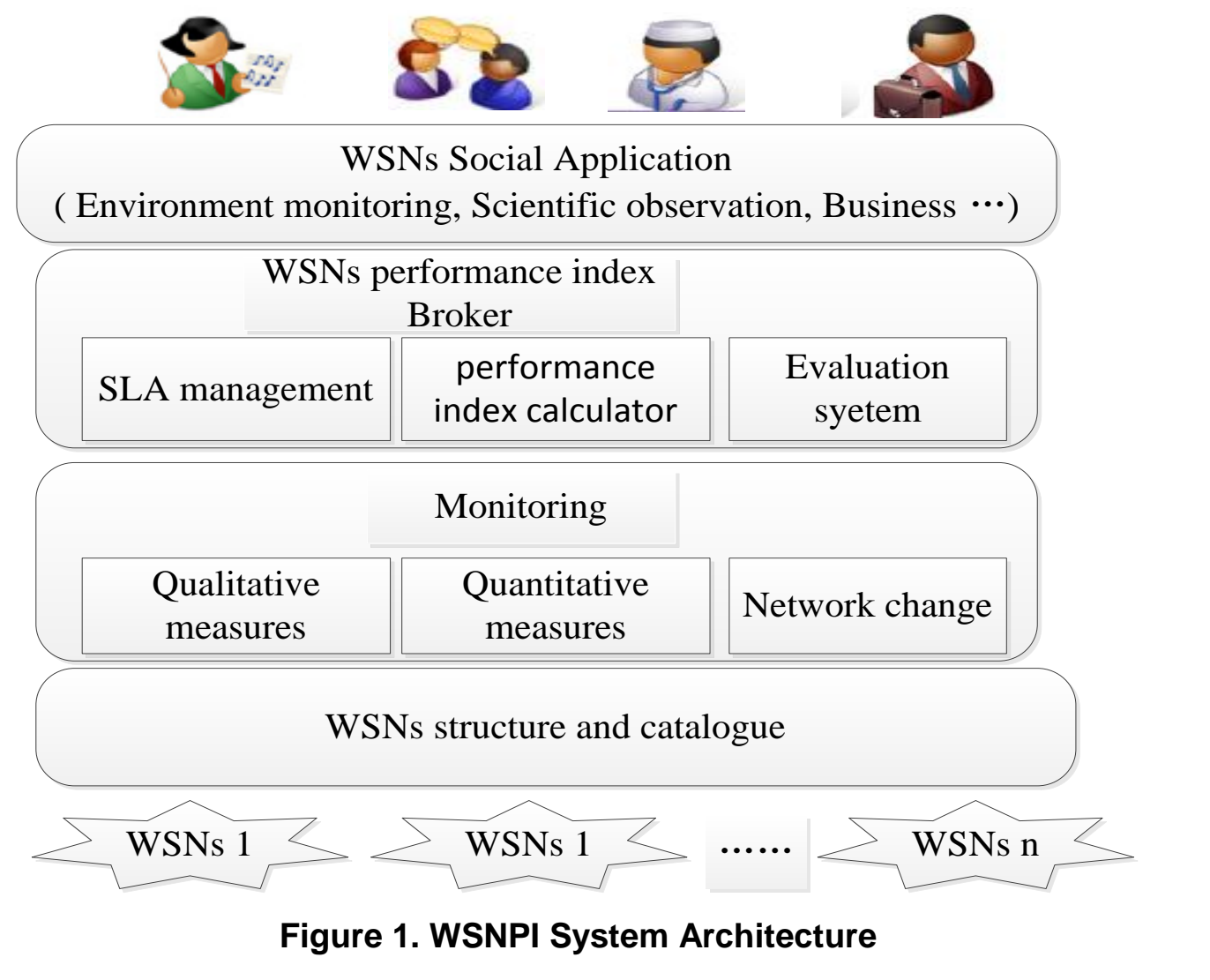

The WSNPI framerork provides features such as WSNs selection based on performance requirements and anking of WSNs based on previous user experiences and real time performance. Figure 1 shows the key elements of the framework:

(1) WSNP Broker: This component is responsible for interaction with customers and understanding their application needs, which performs discovery and ranking of suitable services using other components such as the WSNPI Calculator and Evaluation systems. Evaluation System evaluates WSNs selected by the WSNPI Broker. The WSNPI Calculator computes the various WSNPIs which are used by the ranking system for prioritizing WSNs.

(2) Monitoring: this component first discovers WSNs that can satisfy users' needs. Then, it closely monitors the performance of the WSNs, such as accountability, agility, and cost. For this layer, many tools are available, but some of which we address in the related section.

(3) WSNs Structure and Catalogue: builds the WSNs and their features advertised by various different providers. 
Two important issues in building the framework, as previously mentioned, are the measurement of various WSNPIs and the evaluation of WSNs. In the next section, we put forward a performance evaluation model based on cloud model and entropy weight method.

\section{WSNs Performance Comprehensive Evaluation Model}

The performance evaluation of WSNs is fuzzy and stochastic. Therefore, it is more practical to establish a comprehensive evaluation model by the cloud concept and method than other traditional evaluation methods. In the process of comprehensive evaluation, the weight is the major component, and entropy can reflect the size of the index weight.

\subsection{Cloud Model Theory}

Li firstly proposed the cloud model of qualitative and quantitative on the basis of the traditional fuzzy mathematics and probability statistics. Normal cloud is the most weight cloud model, and the expected curve of normal cloud is a normal curve One dimensional normal digital characteristic of the cloud, $\mathrm{A}=\mathrm{T}(\mathrm{Ex}, \mathrm{En}, \mathrm{He})$ cân be represented by the expected value Ex, entropy En, and super entropy He ju the region [Ex, 3En]. Given the normal cloud characteristic, we can produce the nornal cloud model of 2 dimensions: cloud droplets, called positive cloud generator. The algorithm is as follows.

(1) Generate normal random number $E_{n}^{\prime}=N o$ r m r $\left(E_{n}, H_{v e}\right)$, in which $E_{n}$ is expected value, and $H_{e}$ is variance, Norm rnd $(E, H)$ is a function of normal random number.

(2) Generate normal random number $C_{\text {orm }} \operatorname{rrd}\left(E_{E_{n}}\right)$, in which $E_{x}$ is expected value, and $E_{n}^{\prime}$ is variance.

(3) Calculate certainty degree of

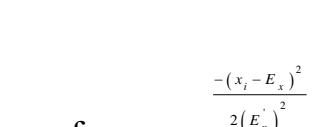

(4)Take $\left(x_{i}, y\right)$ as cload droplets in the domain.

(5) repeat the steps (1)-(4), unti we get $N$ cloud droplets.

\subsection{Methods for Determining Weights Based on Entropy Weight}

At present, researchers usually determined the weight by a subjective method, when they evaluated the network performance of WSNs. Due to the subjective factors, it will cause the evaluation results deviation. In information theory, entropy reflects the degree of disorder of information, therefore, can be used to assess the weight of network performance parameters. The calculation steps are as follows:

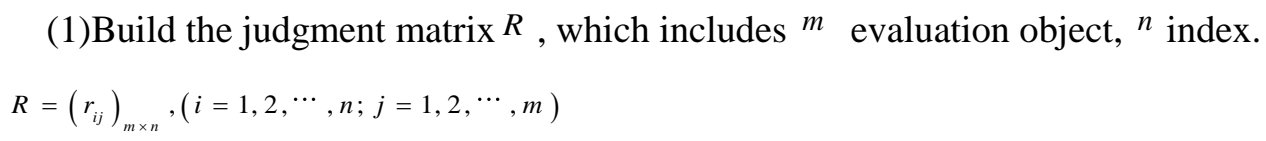

(2) Normalize the judgment matrix $R$, obtain the normalize matrix $B$.

$$
\begin{aligned}
& b_{i j}=\left(r_{\max }-r_{i j}\right) /\left(r_{\max }-r_{\min }\right) \\
& b_{i j}=\left(r_{i j}-r_{\min }\right) /\left(r_{\max }-r_{\min }\right)
\end{aligned}
$$


(3) According to the definition of entropy, we calculate the entropy value of distinct index.

$H_{i}=-\frac{\sum_{j=1}^{m} f_{i j} \ln f_{i j}}{\ln m},(i=1,2, \cdots, n ; j=1,2, \cdots, m)$

In the forma $f_{i j}=b_{i j} / \sum_{j=1}^{m} b_{i j}, 0 \leq H_{i} \leq 1$. If $f_{i j}=0$, then $f_{i j} \ln f_{i j}=0$.

(4)Calculate the entropy weight of evaluation index by using the entropy method, the entropy weight of $i$ index is defined as follows:

$$
w_{i}=\frac{1-H_{i}}{n-\sum_{i=1}^{n} H_{i}}
$$

The bigger the entropy value is, the bigger the entropy weight is, and it shows us that the evaluation index is more critical for more corresponding informaton.

\subsection{Comprehensive Evaluation Model based on Cloud Model and Entropy Weight}

Owing to the characters of dynamic, distribution, resource constrained, uncertainty, complexity and heterogeneity, meanwhile different WSNs applications have different characteristics and requirements in the respective field Therefore, it is more practical to establish a comprehensive evaluation model by the cloud concept and method than other traditional evaluation methods. In the process of comprehensive evaluation, the weight is the fundamental component, and entropycan reflect the size of the index weight. We build an overall evaluation model, which integrates cloudmodel and entropy weight method $t$.

(1) Establish the factordomain of evaluation object $U=\left\{u_{1}, u_{2}, \cdots, u_{n}\right\}$.

(2) Establish thecomments donain ovaluation object $V=\left\{v_{1}, v_{2}, \cdots, v_{m}\right\}$.

(3) Calculate the entropy yeight of evaluation index by using the entropy method, the entropy weight of each in dex is $W=\left\{w_{1}, w_{2}, \cdots, w_{n}\right\}$.

(4) Carry out single factor evaluation between the factor domain $U$ and the comments domain $^{V}$, then establish fuzzy relationship matrix $R \cdot{ }^{r_{i j}}$ is the membership degree of ${ }^{u_{i}}$ to $v_{j}$ in the comments domain ${ }^{V}$. Define each comments interval as bilateral constraints intervar $\left.{ }_{\text {max }}\right]$, calculate the parameters of the half rise cloud and the half fall cloud:

$E_{n}=\left(c_{\text {min }}-d_{1}\right) / 3, x_{i}<E_{x}$

$H_{e}=k_{1}$ 


$$
\left\{\begin{array}{l}
E_{x}=c_{\text {max }} \\
E_{n}=\left(d_{2}-c_{\text {max }}\right) / 3, x_{i}>E_{x} \\
H_{e}=k_{2}
\end{array}\right.
$$

In the forma, ${ }^{d_{1}}$ is the lower range value of good comments in the evaluation factors. ${ }^{d_{2}}$ is the double upper bound of bad comments. ${ }^{k_{1}}$ and ${ }^{k_{2}}$ is experience value given by excerpts.

(5)Comprehensive evaluation model of WSNs performance is synthetic operation, which is equal to weight vector $W$ multiply by $R \cdot C=\left(c_{j}\right)_{1 \times m}=W \circ R, C$ is evaluation result of WSNs performance, $c_{j}=\sum_{i=1}^{N} w_{i} r_{i j}(j=1,2, \cdots, m)$.

(6) The introduction of cloud model, makes the evaluation process different with general fuzzy comprehensive evaluation. Therefore, in order to improve the credibility of evaluation, we need to repeat steps (1)-(5), and calculate of fuzzy evaluation yalue in different membership degree, $C_{i}(i=1,2, \cdots, \mathrm{N})$, finally obtain the average fuzzy/comprehensive evaluation value $C_{\text {final }}=\sum_{i=1}^{N} C_{i} / N$, take $M a x c_{j}$ as the final results and conpute the probability.

\section{A Numerical Example Analysis}

In order to obtain a large number of aceurate real-time data, in this paper, we construct a WSNs simulation environment by using the network simulator NS2. The WSN comprises of 1 network coordinator, 6 routers and 8 terminal nodes. Each terminal node and router can transmit and receive data packets, and can connect with each other. In the simulation run time, randomly generate packets and send through the shortest path to the destination. Performance indicators selected for the network are accountability, agility, cost, actual performance, security and privacy. We evaluate the performance of wireless sensor network based on ZigBee technology to build different networks. The simulation is performed in the laboratory including common software and hardware environment, namely CPU Intel core $4.0 \mathrm{GHz}$, menory for the DDRJ $4 \mathrm{G}$, operating system is Windows7.0 professional edition.

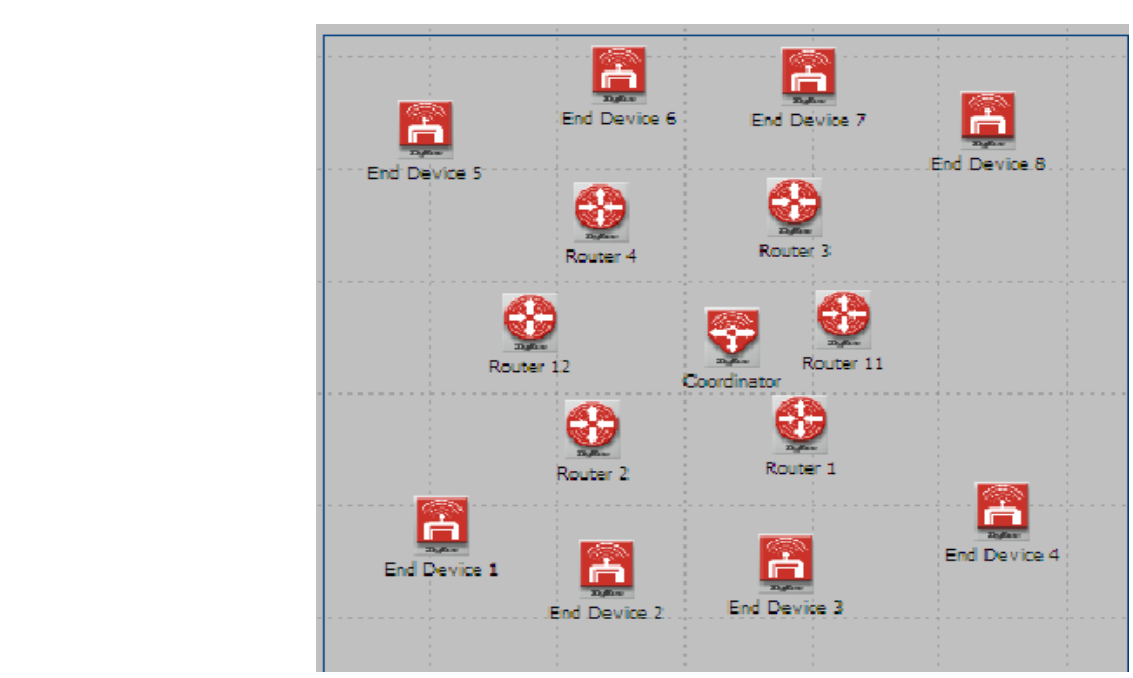

Figure 2. WSNs Simulation Environment 


\subsection{Sample Data}

The data of 10 WSNs collected by simulation are shown in Table 1 . These factors are divided into two types. Factors $\mathrm{x} 1$ to $\mathrm{x} 4$ belong to the type in which the bigger of the value the better. Factor $x 5$ belongs to the type in which the smaller of the value the better.

Table 1. Statistical Practical Data of WSNs

\begin{tabular}{|c|c|c|c|c|c|c|}
\hline \multirow{2}{*}{$\begin{array}{c}\text { Number of } \\
\text { WSNs }\end{array}$} & \multirow{2}{*}{$\begin{array}{l}\text { Accountability } \\
\qquad\left(x_{1}\right)\end{array}$} & \multicolumn{2}{|c|}{ Actual performance $\left(x_{2}\right)$} & \multirow{2}{*}{$\operatorname{Agility}\left(x_{3}\right)$} & \multirow{2}{*}{$\begin{array}{c}\text { Security and } \\
\operatorname{privacy}\left(x_{4}\right)\end{array}$} & \multirow{2}{*}{$\operatorname{Cost}\left(x_{5}\right)$} \\
\hline & & $\begin{array}{c}\text { Time } \\
\text { delay }\left(x_{21}\right)\end{array}$ & $\begin{array}{c}\text { Packet loss } \\
\operatorname{rate}\left(x_{22}\right)\end{array}$ & & & \\
\hline 1 & 5 & 0.01235 & 0.00896 & 11.6 & 6 & 8 \\
\hline 2 & 6 & 0.05864 & 0.00256 & 7.9 & 8 & 7 \\
\hline 3 & 4 & 0.08745 & 0.00357 & & & 128 \\
\hline 4 & 5 & 0.01235 & 0.04785 & & & 114 \\
\hline 5 & 3 & 0.00854 & & & 6 & 87 \\
\hline 6 & 8 & 0.00457 & & -20. & 8 & 120 \\
\hline 7 & 8 & 0.05748 & & 19 & 6 & 113 \\
\hline 8 & 4 & 0.0075 & & 11.3 & 4 & 98 \\
\hline 9 & 6 & & 0.00452 & 9.6 & 4 & 89 \\
\hline 10 & 9 & 000 & 0.03068 & 8.3 & 8 & 102 \\
\hline
\end{tabular}

\subsection{Calculation of Entropy}

In the simulation experiment, simulate different types and operation states of WSNs, use these parameters value under different states to calculate the index weight. In a real network environment, it is different to obtain accurate parameters of WSNs. In the actual application, we should also use of simulation method to obtain the samples to calculate weight. Entropy is calculated according to parameter values in Table 2 and the entropy weight method in Section 2.2, as showed in Table 3. We can see clearly that actual performance is the most important index among all; agility is the least significant index.

Table 2. Indexes Entropy and Weight

\begin{tabular}{|c|c|c|}
\hline Performance indexes & Entropy value & Weight value \\
\hline Accountability & 0.876215 & 0.2024 \\
\hline Actual performance & 0.892230 & 0.3417 \\
\hline Agility & 0.953167 & 0.1175 \\
\hline Cost & 0.942568 & 0.1354 \\
\hline Security and privacy & 0.881452 & 0.2030 \\
\hline
\end{tabular}




\subsection{Comprehensive Evaluation and Analysis of the Results}

From Table 2, the complex WSNs performance is closely linked with factors such as accountability, agility, cost, actual performance, security and privacy. By the methods of Section 2.3, we can obtain half cloud model for each index of different comments and weight. Follow the steps, we continue 1000 times random evaluations and get a result of WSNs 2 for example: $\mathrm{C}=(0.9754,0.3345,0.1049)$. In the 1000 calculation, membership degree of good comments is always higher than that of bad. Therefore, the assessment result is excellent with probability $100 \%$, reflects the effects of different experts on the evaluation results by probability value, and makes the results more credible. Finally, a list of comprehensive evaluation values and rankings is shown in Table 3.

Table 3. Comprehensive Evaluation Values and Rankings

\begin{tabular}{|c|c|c|}
\hline Number of WSNs & Comprehensive evaluation values & \\
\hline 1 & $\mathrm{C}=(0.8874,0.4398,0.0456)$ & \\
\hline 2 & $\mathrm{C}=(0.9821,0.3058,0.1274)$ & 1 \\
\hline 3 & $\mathrm{C}=(0.7426,0.2589,0.4035)$ & 9 \\
\hline 4 & $\mathrm{C}=(0.7235,0.4263,02034)$ & 10 \\
\hline 5 & $\mathrm{C}=(0.9687,0.4125,0.2145)$ & 3 \\
\hline 6 & $\mathrm{C}=(0.9768,0.3874,0.1354)$ & 2 \\
\hline 7 & $\mathrm{C}=(0.8752,0.3541,0.3546)$ & 6 \\
\hline 8 & $\mathrm{C}=(0.7563,0.3024,0.3357)$ & 8 \\
\hline 9 & $\mathrm{C}=(0.7982,0.3204,0.3542)$ & 7 \\
\hline 10 & $\mathrm{C}=(0.9654,0.3354,0.2457)$ & 4 \\
\hline
\end{tabular}

\section{Conclusion}

Last several years, with the rapid development of embedded computing technology, modern network, and wireless communication technology, wireless sensor network has a very vast application prospect and potential utility value, which integrates a large number of relat ve technology together. In most of WSN applications, a large number of sensor nodes are deployed to gather data based on application domains. WSNs opens up a wealth of opportunities for different applications, such as agriculture, city management, environmental monitoring, health management, emergency rescue and disaster relief, anti-terrorism, remote control of hazardous area and so on. Due to the characters of dynamic, distribution, resource constrained, uncertainty, complexity and heterogeneity, we need a whole evaluation framework of WSNs performance, which can help users choose and monitor the operation state. In this paper, we construct WSNPI architecture to rank and observe WSNs, which includes WSNPI Broker, Monitoring and WSNs Catalogue. We propose a more 
comprehensive evaluation index system including accountability, agility, cost, security and privacy, then build a cloud model and entropy weight method to evaluate WSNs performance. Finally, the proposed method has been used in complex simulation, and the results have indicated that the model is unbiased and effective. The factors to consider in this study can't be all covered, that is to say, the evacuation model now established as a starting point, there are many issues and details need to be more in-depth study and discussion.

\section{Acknowledgements}

The authors gratefully acknowledge the financial support from China National Natural Science Fund of China (61302124), Natural Science Fund of Jiangsu Province (BK20130235), Natural Science Foundation of the Higher Education Institutions of Jiangsu Province (13KJB520006), Program of six talent tops of Jiangsu Province (DZXX-031).

\section{References}

[1] M. Keshtgari and A. Deljoo, "A Wireless Sensor Network Solâtion for Precision Agriculture Based on ZigBee Technology", Wireless Sensor Network, vol. 4, no. 1, (2012).

[2] S. Silva Pereira, "Mean square convergence of consensus algorthms in random WSys. Signal Processing", IEEE Transactions, vol. 58, no. 5, (2010), pp. 2866-2874.

[3] D. A. Swyt, an Assessment of the United States Measurement System: Addressing Measurement Barriers to Accelerate Innovation, 2007 NIST Special Publication 1048.

[4] W. M. Healy and W. S. Jang, "Practical Challenges in Wireless Sensor Network Use in Buildings", 2008 NIST Technical Note 1604.

[5] W. M. Healy, W. S. Jang, Practical Challenges in Wireless Sensor Network Use in Buildings, 2008 NIST Technical Note 1604.

[6] O. Selcuk, D. Karaboga and C. Ozturk, "Ân application of wireless sensor network routing based on artificial bee colony algorithm”, Evolutionary Computation (CEC), 2011 IEEE Congress on. IEEE, (2011).

[7] B. Bhuyan, "Quality of Service (QoS) Provisions In Wireless Sensor Networks and Related Challenges", Wireless Sensor Network, vol. 2, no 1, (2010)

[8] M. Dohler, "The ARESA project: Facilitating research, development and commercialization of WSNs", Sensor, Mesh and Ad Hoc Communications and Networks, 2007. SECON07. 4th Annual IEEE Communications Societ Conference on. IEEE, (2007).

[9] L. Ravindranath, "Improving wireless network performance using sensor hints", Proceedings of the 8th USENIX conference on Networked systems design and implementation. USENIX Association, (2011).

[10] E. Stavrou and A. Pitsillides, "A survey on secure multipath routing protocols in WSNs", Computer Networks, vol. 54, no. 13,(2010), pp. 2215-2238.

[11] W. Y. Poe, M Beck and J. B. Schmitt, "Planning the trajectories of multiple mobile sinks in large-scale, time-sensitive WSNs", Distributed Computing in Sensor Systems and Workshops (DCOSS), 2011 International Conference on. IEEE, (2011).

[12] R. A. Sahner, K. Trivedi and A. Puliafito, "Performance and reliability analysis of computer systems: an example-based approach using the SHARPE software package", Springer Publishing Company, Incorporated, (2012).

[13] P. Wright, D. Dornfeld and N. Ota, "Condition monitoring in end-milling using wireless sensor networks (WSNs)", Transactions of NAMRI/SME36, (2008), pp. 177-183.

[14] J. Peng, R. Han and S. Mishra, "A performance evaluation of intrusion tolerant routing in wireless sensor networks", Information Processing in Sensor Networks. Springer Berlin Heidelberg, (2003).

[157 D. Wang, "Building Wireless Sensor Networks (WSNs) by Zigbee Technology", Journal of Chongqing university (natural science edition), vol. 8, (2006): 023.

[16] R. Silva, J. Sá Silva and F. Boavida, "Evaluating 6lowPAN implementations in WSNs", Proceedings of 9th Conference Computer adores, Portugal, (2009), pp. 1-5.

[17] D. Noh, I. Yoon and H. Shin, "Low-Latency Geographic Routing for Asynchronous Energy-Harvesting WSNs", Journal of Networks, vol. 3, no. 1, (2008).

[18] H. Zhu, I. D. Schizas and G. B. Giannakis, "Power-efficient dimensionality reduction for distributed channel-aware kalman tracking using WSNs", Signal Processing, IEEE Transactions, vol. 57, no. 8, (2009), 3193-3207. 
[19] H. Chen, "Agent-based trust model in wireless sensor networks", Proceedings of the Eighth ACIS International Conference on Software Engineering, Artificial Intelligence, Networking, and Parallel/Distributed Computing, IEEE Computer Society, vol. 03, (2007).

[20] F. Viani, "Pervasive remote sensing through WSNs", Antennas and Propagation (EUCAP), 2012 6th European Conference, IEEE, (2012).

[21] S. Silva Pereira, "Mean square convergence of consensus algorithms in random WSNs", Signal Processing, IEEE Transactions, vol. 58, no. 5, (2010), pp. 2866-2874.

[22] M. Dohler, "The ARESA project: Facilitating research, development and commercialization of WSNs. Sensor, Mesh and Ad Hoc Communications and Networks", 2007. SECON07. 4th Annual IEEE Communications Society Conference, IEEE, (2007).

[23] M. Gholami, N. Cai and R. W. Brennan. "An artificial neural network approach to the problem of wireless sensors network localization." Robotics and Computer-Integrated Manufacturing 29.1 (2013): 96-109.

[24] S. Okdem, D. Karaboga and C. Ozturk, "An application of wireless sensor network routing based on artificial bee colony algorithm”, Evolutionary Computation (CEC), 2011 IEEE Congress, IEEE, (2011).

[25] B. Bhuyan, "Quality of Service (QoS) Provisions in Wireless Sensor Networks and Related Challenges", Wireless Sensor Network, vol. 2, no. 11, (2010).

[26] R. Kumar and S. Goyal, "Perspective of WSNs simulators", Int. J. Inf. Eng. i10 2, (2013), pp 3744

[27] W. Yi Poe, M. Beck and J. B. Schmitt, "Planning the trajectories of multiple mobile ink In large-scale, time-sensitive WSNs", Distributed Computing in Sensor Systems and Workshops (DCOSS), 2011 International Conference on. IEEE, (2011).

[28] R. A. Sahner, K. Trivedi and A. Puliafito, "Performance and rellability analysis of computer systems: an example-based approach using the SHARPE software package" Springer Publishing Company, Incorporated, (2012).

[29] D. Wang, "Building Wireless Sensor Networks (WSNs) by Zigbee Technology", Journal of Chongqing university (natural science edition), vol. 8, (2006), pp. 023.

[30] R. Silva, J. Sá Silva and F. Boavida, "Evaluating 6lowPAN implementations in WSNs", Proceedings of 9th Conference Computer adores, Portugal, (2009), pp. 1-5.

[31] D. Noh, I. Yoon and H. Shin, "Low-Latency Geographic Royting for Asynchronous Energy-Harvesting WSNs", Journal of Networks, vol. 3, no. 18(2008).

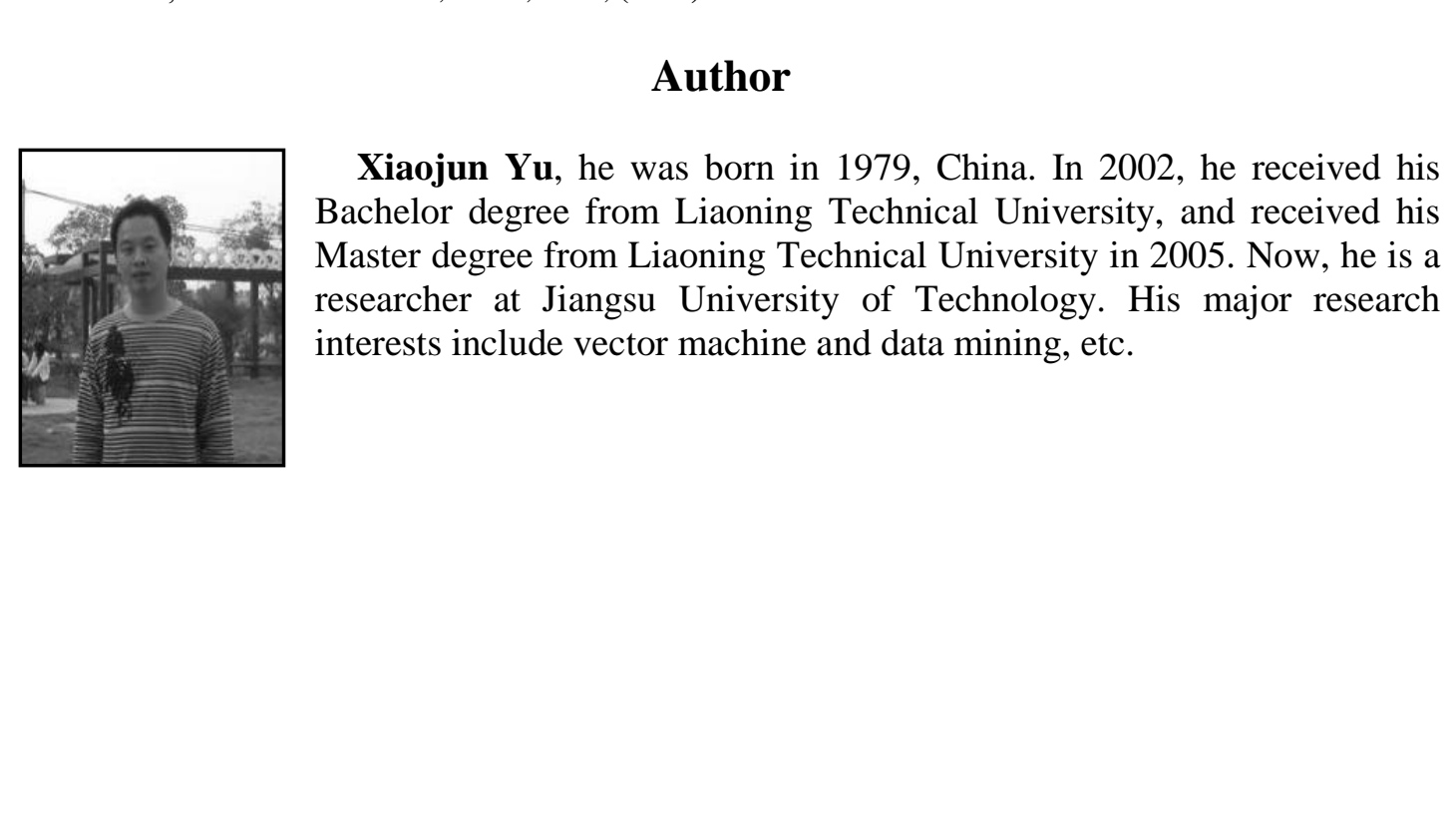

